\title{
Evaluación del uso de la plataforma Moodle en la gestión del aprendizaje universitario en la Facultad de Ingeniería de Sistemas de la UNCP
}

\author{
Evaluation of the use of the Moodle platform in the management of \\ university learning in the Faculty of Systems Engineering of the UNCP
}

\author{
aHuaman Samaniego, H.; Vásquez Caicedo, M.; Santivañez Esquivel, I. \\ Facultad de Ingeniería de Sistemas / Universidad Nacional del Centro del Perú \\ Email: hhuaman@uncp.edu.pe
}

\section{Resumen}

El trabajo plantea el problema de la influencia del uso de la plataforma Moodle en la gestión del aprendizaje universitario en la Facultad de Ingeniería de Sistemas de la UNCP, con el objetivo de poder establecer la relación del uso con la enseñanza aprendizaje, de manera que se puedan identificar algunas estrategias para reforzar el trabajo virtual, como soporte en la formación profesional.

La investigación es descriptiva, de diseño no experimental transaccional, asumiendo la aplicación de un instrumento que mide el uso de la plataforma en tres dimensiones en particular: lo general, la navegación y diseño y el uso de las herramientas multimedia.

Se determinó que, el $58 \%$ de los docentes registran uso de la plataforma virtual y a manera de repositorio, sin que sea importante para reforzar la formación estudiantil. El $23.3 \%$ de los docentes, no hacen uso alguno de la plataforma virtual y, predominantemente, corresponden a los cursos cuánticos, como: matemática, estadística, física, entre otros. La dimensión de la calidad general y de la metodología, que es la que mejor apreciación tiene tanto docente como estudiantil, y la dimensión de la calidad técnica de los recursos multimedia, es la que menos preferencia representa por parte de los docentes, debido fundamente a la poca accesibilidad y adaptabilidad a dichos recursos.

Palabras clave: plataforma virtual, gestión de aprendizaje, enseñanza universitaria, TIC

\begin{abstract}
The research raises the problem of the influence of the use of the Moodle platform in the management of university learning in the Faculty of Systems Engineering of the UNCP, with the aim of being able to establish if there is any relation of use with teaching and learning, so that some strategies can be identified to reinforce virtual work as a support in professional training.

The research is descriptive. The design is non-experimental transactional, assuming the application of an instrument that measures the use of the platform in three dimensions in particular: the general, navigation and design and the use of multimedia tools.

The main findings are that only $58 \%$ of teachers register use of the virtual platform and as a repository, without it being important to reinforce student training. 23.3\% of teachers do not make use of the virtual platform, and predominantly correspond to quantum courses, such as: mathematics, statistics, physics, among others. The dimension of the general quality and of the methodology that is the one that has the best appreciation both the teacher and the student and the dimension of the technical quality of the multimedia resources, is the one that represents the least preference on the part of teachers, due priority to poor accessibility and adaptability to these resources.
\end{abstract}

Keywords: virtual platform, learning management, university teaching, ICT 


\section{Introducción}

Para los procesos de enseñanza aprendizaje, se presentan diversas estrategias pedagógicas, entre las cuales, los entornos virtuales se han convertido en una alternativa viable y asertiva para la nueva generación, en el marco constructivista de la educación. La capacidad interactiva y visual que permiten el uso de las tecnologías de comunicación e información, se adecúan a las necesidades de un entorno agradable para enseñar y aprender. Todo dependerá del grado de imaginación y habilidad del docente para enseñar y del estudiante para aprender, pero de manera interactiva, donde el docente también podrá aprender de las contribuciones del estudiante, al utilizar ambos utilizan las plataformas tecnológicas de información y comunicación.

La enseñanza aprendizaje es una tarea que necesariamente implica al docente y estudiante, en una interacción permanente que debe conducir al mejor aprovechamiento de las TIC, pero orientado a las metas, objetivos y, sobre todo, a mejorar las competencias de los estudiantes. Por lo tanto, para que puedan aprovechar mejor los estudiantes, se requiere que el docente muestre capacidades suficientes en el uso de la plataforma virtual.

Si bien se disponen de plataformas virtuales, el problema se centra ahora en el uso; por ello, el trabajo pretende evaluar el uso de la plataforma por los docentes de la Facultad de Ingeniería de Sistemas de la UNCP, para poder evidenciar en cuánto se utilizan las tecnologías de información y comunicación como apoyo en el desarrollo de su labor académica.

\section{Métodos y materiales}

El tipo de investigación es descriptivo, al dimensionar el uso de la plataforma Moodle como herramienta de enseñanza aprendizaje y evaluar su influencia en la gestión del aprendizaje universitario; sin embargo, se desestimó trabajar la correlación, al no disponerse de evidencia de la gestión del aprendizaje. Se utilizó el método inductivo y deductivo. Las fuentes de información son: el informe de la plataforma por parte del administrador y; para disponer de la percepción del uso, se aplicó una encuesta para evaluar la utilización del Moodle, que contempla tres dimensiones. La investigación se desarrolló en la Facultad de Ingeniería de Sistemas, incluyendo a docentes y estudiantes, respondiendo al diseño transaccional. Para este caso se considera dos semestres, impar y par del año 2018. Al instrumento se calculó la fiabilidad, alcanzado un alfa de Cronbach de 0.982, que significa un alto grado de fiabilidad del instrumento. Se utilizó el software del SPSS en su versión 24, para el procesamiento estadístico de la información.

\section{Resultados}

La mejora en el uso de la plataforma virtual es evidente, pero no se puede afirmar categóricamente que así sucedió, por cuanto se requiere mayor información de más períodos, pero no anteriores, sino futuros, ya que la muestra del uso del Moodle fue menor en los años anteriores (Figura 1).

\section{Figura 1}

Uso de la plataforma virtual comparada

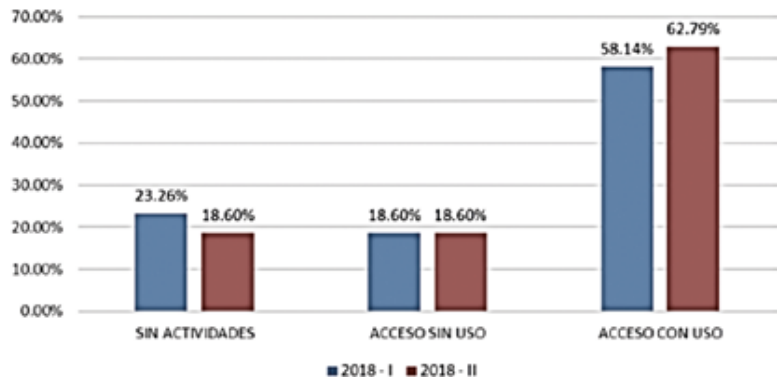

La sostenibilidad en el uso de la plataforma, se tendría que evaluar para el futuro, por asignatura, docente e incluso estudiantes. Se trabajaron tres dimensiones:

\section{Calidad general del entorno y de la metodología}

La dimensión es todavía muy genérica, pero no le resta la importancia de calcular apreciaciones, como se muestra en la Figura 2.

\section{Figura 2}

Dimensión general del entorno de la plataforma y la metodología

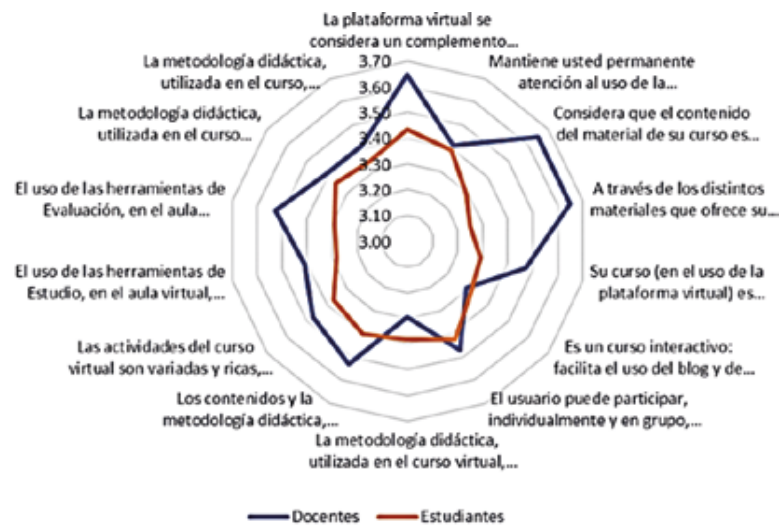

Los puntajes no son tampoco altamente significativos, pero si marcan una clara diferencia entre la apreciación docente y estudiantil. Teniendo en cuenta la escala de Likert, tienen gran porcentaje el nada, algo y poco; que marcan una relativa y considerable moderación en la calificación, por lo que estar más de la mitad, significa un poco de utilidad y uso de la plataforma. No significa que se está bien, que por lo menos sería lo ideal.

Hace falta trabajar más los criterios metodológicos, para que el estudiante pueda realmente sentir una ayuda en su aprendizaje y que el docente se apoye en herramientas que hasta ahora son poco conocidas para él. Es un proceso de adaptabilidad a un nuevo esquema en el que los docentes se vienen adecuando, por ser de una época y generación anterior, que en su mayoría no crecieron con un computador y menos articulados a la Internet. 


\section{Calidad técnica de navegación y diseño}

Existen algunos aspectos de coincidencia, como el caso de la plataforma Moodle, que presenta herramientas de comunicación, evaluación y estudio de calidad, donde los puntos de vista docente y estudiantil, suelen coincidir en valoración en cuanto se refiere a la apreciación.

Esta similitud de apreciación es pertinente, si se tiene en cuenta que es el único software que se utiliza para el aula virtual; posiblemente, si se utilizará otras plataformas, las apreciaciones serían diferentes.

Otro punto casi coincidente, se refiere al tamaño de los íconos y botones, sobre si son adecuados, que para los estudiantes obviamente que sí, mientras que para los docentes la magnitud es menor (ver Figura 3).

\section{Figura 3}

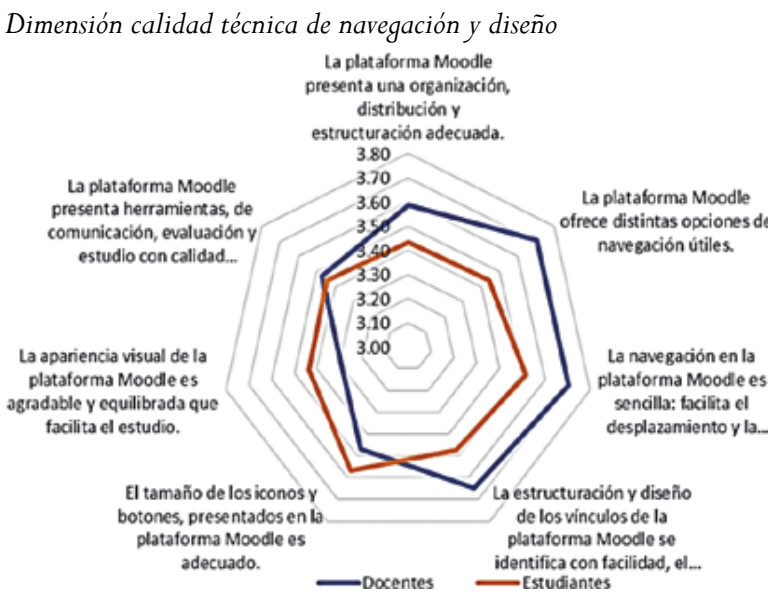

Los aspectos de relativa coincidencia, pero marcada diferencia cuantitativa, están referidos a la organización y distribución adecuada de la plataforma. La pregunta de que se ofrece distintas opciones de navegación útiles y que la navegación es sencilla en la plataforma Moodle, el gráfico puede evidenciar el mismo sentido, pero marcada diferencia en la apreciación docente y estudiantil.

Los estudiantes suelen considerar que los tamaños de los íconos son adecuados en una mayor proporción que los docentes, quienes consideran su relativa baja puntuación, debido a la familiaridad que tienen los estudiantes con los celulares y suelen navegar con mayor facilidad y asocian los íconos de una y otra herramienta, que cada vez se universaliza.

Las propiedades de los íconos en un aplicativo u otro, incluso en los video juegos, son casi similares, por lo que de cualquier manera, los estudiantes suelen adaptarse mejor; mientras que los docentes, por su propia naturaleza de haber nacido en una diferente época y poca asociación con los aplicativos y herramientas, no son pasibles de fácil adaptación en el uso de íconos y se prefiere ventanas y accesos directos de mayor interacción.

\section{Calidad técnica de los recursos multimedia}

En ésta dimensión, por la naturaleza de la orientación de las preguntas a los recursos multimedia en sí, no se tienen respuestas significativas; es decir, que los puntajes de apreciación suelen ser menores, tanto para los docentes como para los estudiantes (Figura 4).

\section{Figura 4}

Dimensión calidad técnica de los recursos multimedia

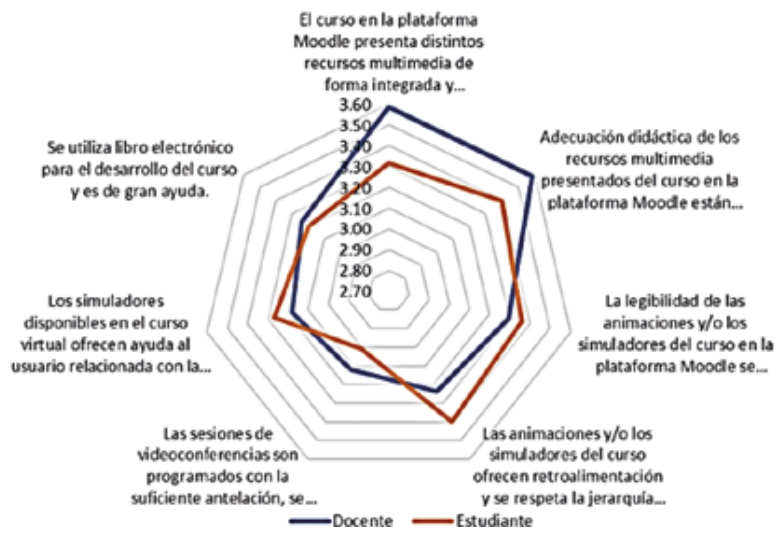

Las evaluaciones más bajas, están referidas al uso de los textos electrónicos, que no es costumbre aún utilizar un texto base, que sirva de referencia y soporte al desarrollo de la asignatura. El docente trata de mantener algún texto que el estudiante no sepa y el estudiante trata de encontrar dicho texto en una permanente búsqueda de solución de ejercicios para continuar de manera repetitiva en el desarrollo de la asignatura.

Así mismo, se tiene menor grado de apreciación el uso de las video conferencias, que es otra costumbre poco utilizada en la plataforma. Los estilos tradicionales persisten, de sólo dictar la "conferencia" por parte del docente en el aula, dejando que el estudiante, resuelva o exponga casos también en el aula, sin utilizar herramientas tecnológicas.

Una de las razones es que el docente no tiene el tiempo suficiente para preparar clases, menos aún para un video conferencia, que requiere de mayor dedicación y uso de otras herramientas complementarias, a las cuales tampoco tiene acceso. Comparativamente se pueden apreciar las tres dimensiones (ver fígura 5).

\section{Figura 5}

Comparación de las tres dimensiones de uso

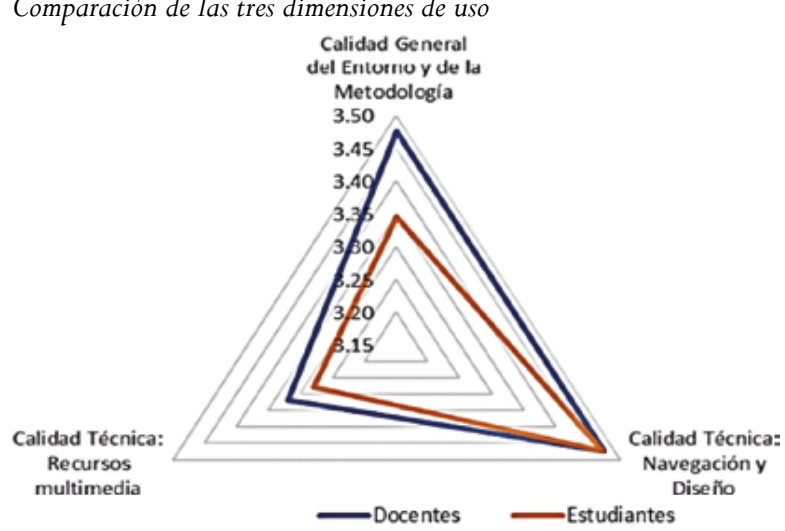


Como se indicó, las valoraciones son muy moderadas, partiendo de considerar que no se utilizan nada, poco y algo, que en suma nos presenta un panorama de poca exigencia en el uso de la plataforma y esto se debe a la no existencia de patrones definidos, pedagógicamente, de exigencias mínimas que pueden normar el desarrollo de todas las asignaturas, independientemente del número de horas, tipo de curso, entre otras razones.

No se puede considerar que se está bien, los puntajes son muy bajos, pero se está acercando a lo favorable en cuanto al uso de herramientas virtuales, como se muestra en la Figura 6.

\section{Figura 6}

Comparación de las tres dimensiones de uso

\begin{tabular}{|c|c|c|c|c|c|c|}
\hline \multicolumn{2}{|c|}{ DIMENSIONES } & $\begin{array}{c}1 \\
\text { Nada }\end{array}$ & $\begin{array}{c}2 \\
\text { Murpoco }\end{array}$ & $\begin{array}{c}3 \\
\text { Ago }\end{array}$ & $\begin{array}{c}4 \\
\text { Bestante }\end{array}$ & $\begin{array}{c}5 \\
\text { Mucto }\end{array}$ \\
\hline \multicolumn{7}{|c|}{ Calidad General del Entorno y de la Melodologia } \\
\hline $\begin{array}{c}\text { Docente } \\
\text { Estudlante }\end{array}$ & $\begin{array}{l}3.47 \\
3.34\end{array}$ & & & & & \\
\hline \multicolumn{7}{|c|}{ Caildad Técrica: Nevegagaión y Diseño } \\
\hline $\begin{array}{c}\text { Docante } \\
\text { Estudiate }\end{array}$ & $\begin{array}{l}3.47 \\
3.47\end{array}$ & & & & & \\
\hline \multicolumn{7}{|c|}{ Calisad Técrica: Recursos mulinedia } \\
\hline $\begin{array}{c}\text { Docente } \\
\text { Estudiante }\end{array}$ & $\begin{array}{l}3.32 \\
3.28 \\
\end{array}$ & & & & & \\
\hline
\end{tabular}

\section{Discusión}

Encontrar nuevas estrategias de enseñanza aprendizaje, se convierte en tareas ahora complicadas y de difícil implementación, al requerirse, cada vez, cambiar paradigmas y romper viejas ataduras de la tradicional enseñanza. Al respecto, el $35 \%$ de los docentes y el $47 \%$ de los estudiantes, reconocen que la plataforma Moodles es un complemento muy significativo, a los materiales impresos en el desarrollo de las clases, convirtiéndose en una principal fuente de apoyo a diferencia de los tradicionales mecanismos de enseñanza. (Glasserman et al., 2014).

El trabajo evidencia que aún es incipiente el uso de tecnologías de información y comunicación en la Facultad de Ingeniería de Sistemas de la UNCP, no se llega al nivel de bastante, en el uso y; se es muy moderado en la evaluación del uso, al considerar sólo algunos elementos básicos. Se han registrado evidencias del uso de la plataforma a manera de repositorio y no precisamente para orientarlo a la enseñan aprendizaje, que puede estar asociado al tema de la edad de los docentes u otros mecanismos de inducción necesarios.

Entonces, estará en cuestionamiento si los resultados de la evaluación miden precisamente el uso de la plataforma de manera eficaz y tendremos la discusión de lo presencial y lo virtual, para continuar en el proceso de cuestionamiento al apoyo virtual. (Cebreiros, 2011).

No cabe duda que la plataforma Moodle permite mejorar las capacidades de enseñanza aprendizaje, pero aún se evidencian necesidades de implementar estrategias didácticas propias, que tengan un fin en particular y merezcan la debida preparación, tanto en docentes y estudiantes, a pesar de ser el Moodle una plataforma sin complicaciones, ágil y adaptable a toda la comunidad estudiantil y docente (Muñoz, Gómez, \& Alemán, 2016).

Mientras que el $58.8 \%$ de los estudiantes considera que las animaciones ayudan en algo el desarrollo académico, el $41.7 \%$ de los docentes también así lo consideran, diferencia que es comprensible por la no existencia de archivos que ayuden el desarrollo académico de los estudiantes; así, si no existen no ayuda, pero para los docentes el prepararlos implicaría más tiempo y dedicación, que puede ser desarrollado a través de una buena clase presencial, a la que suelen darle mayor importancia, restándole la importancia a virtualizar las clases y el proceso de enseñanza aprendizaje, no se seleccionan materias, ni temas apropiados para desarrollarlos de manera virtual, ni se asignan tareas efectivas por este medio (Fariña, González, \& Area, 2012).

Los docentes le dan mayor prioridad e importancia a las clases presenciales y el uso de la plataforma Moodle se considera para las clases virtuales; de allí que, por ejemplo, el uso de textos virtuales en las clases para los estudiantes es muy importante, alcanzando hasta un 47 \% comparado con el $25 \%$ de los docentes en el nivel de importancia. Así, el desarrollo del b-learning, que simplifica la expresión del blended learning y la preparación en línea, se convertirá en una fuerte limitación, tanto por concepto, criterio y uso (Morales, 2012).

Utilizar la plataforma a manera de interactuar con los estudiantes, es aún incipiente, se prefiere dejar las clases como evidencia de su uso del Moodle, pero no se trabaja en el uso de herramientas tecnológicas basados en TICs que pueden mostrar procesos de mejora de la calidad de la educación. (Gámiz, 2009).

El $23.5 \%$ de los estudiantes, considera que la navegación en el Moodle es de fácil accesibilidad, mientras que el $15 \%$ de los docentes así lo consideran. Esto refiere el tema de la edad de los docentes, que aún no se adaptan a las herramientas compartidas que tienen muchas de las utilidades, entre ellas, las posibilidades del Moodle de articularse a otros softwares de colaboración comunitaria. Falta reafirmar y utilizar más aplicaciones que pueden reforzar la enseñanza aprendizaje, empezando por sus propios cursos, tareas y usos de aplicaciones y herramientas (Estrella, 2012).

Los docentes, son los que menos se adaptan al uso del Moodle y hay mayor grado de resistencia al uso de la plataforma, poco más de la mitad, han registrado cierto uso del aula virtual ( $58 \%$ ); sin embargo, es necesario comprender que las TIC van a mejorar el rendimiento de los estudiantes si utilizan nuevas tecnologías. (Rodríguez, 2010). 


\section{Conclusiones}

- Sólo el 58 \% de los docentes registran uso de la plataforma virtual y no necesariamente para reforzar su proceso de enseñanza aprendizaje, al tenerse, a manera de repositorio, la plataforma virtual, sin que sea importante para reforzar la formación estudiantil.

- El $23.3 \%$ de los docentes no hacen uso alguno de la plataforma virtual, que predominantemente corresponden a los cursos cuánticos, como: matemática, estadística, física, entre otros, que se asocia como de poca importancia el uso de la plataforma virtual y no aprovechan las múltiples herramientas del sistema.

- Durante el semestre par 2018, alcanzó el $62.8 \%$ de docentes que tienen acceso a la plataforma, incrementándose la participación, sin que se registre un real y efectivo uso que apoye la enseñanza aprendizaje, no hay evidencias de mejoras en la parte pedagógica; por lo tanto, no es posible afirmar resultados en formación profesional con el uso de la plataforma.

- La dimensión de la calidad general y de la metodología, que es la que mejor apreciación tiene, tanto docente como estudiantil, no llega al nivel de un uso de bastante, de manera categórica; por lo que, a pesar de ser la dimensión mejor valorada se mantienen necesidades de mejora en todos los niveles, pedagógicos, de inducción, control y seguimiento al uso efectivo de la plataforma.

- La dimensión de la calidad técnica de los recursos multimedia, es la que menos preferencia representa por parte de los docentes, debido fundamente a la poca accesibilidad y adaptabilidad a dichos recursos, lo que no sucede con los estudiantes que muestran mayor predisposición a su uso.

\section{Referencias bibliográficas}

Agencia de Gobierno Electrónico y Sociedad de la Información y el Conocimiento. (2012). eParticipación: conceptos básicos y buenas prácticas. Montevideo, Uruguay: Agesic.

Barbera, E. (2006). Aportaciones de la tecnología a la e-Evaluación. Estudios de Psicología y Ciencias de la Educación. Revista de Educación a distancia, 1-13.

Cebreiros, E. (2011). La plataforma Moodle y la evaluación de aprendizajes. II Jornadas sobre docencia del derecho y tecnologías de la información y la comunicación, 1 - 8.

Cobarrubias, P. \& Piña, M. (2004). La interacción maestro-alumno y su relación con el aprendizaje. Revista Latinoamericana de Estudios Educativos, México, vol. XXXIV, 47-84.
Doménech, F. \& García, F. (2002). Motivación, aprendizaje y rendimiento escolar. Docencia, No 16, 1-13.

Estrella, A. (2012). Uso de la plataforma tecnológica Moodle por docentes de primaria del Instituto Irlandés Masculino de México: Un Análisis con énfasis en la mejora de la educación actual. Edición Única. Estado de México: Tecnológico de Monterrey, Universidad Virtual.

Fariña-Vargas, E.; González-González, C. \& AreaMoreira, M. (2012). ¿Qué uso hacen de las aulas virtuales los docentes universitarios? RED. Revista de Educación a Distancia. Número 35, 1-13.

Foresti, P. \& Goncalves, P. (2010). Sociedade da informação e o uso da tecnologia. Revista das Faculdades Integradas Claretianas. No 3, 1-18.

Gallardo, G. \& Reyes, P. (2010). Relación profesor alumno en la universidad: Arista fundamental para el aprendizaje. Calidad en la Educación, 1-31.

Gámiz, V. (2009). Entornos virtuales para la formación práctica de estudiantes de educación: Implementación, experimentación y evaluación de la plataforma Aulaweb. España: Universidad de Granada.

Garbanzo, G. (2013). Factores asociados al rendimiento académico en estudiantes universitarios desde el nivel socioeconómico: Un estudio en la Universidad de Costa Rica. Revista Electrónica Educare, 1-31.

García-Beltrán, A.; Martínez, R.; Jaén, J. A. \& Tapia, S. (2016). La autoevaluación como actividad docente en entornos virtuales de aprendizaje/enseñanza. RED. Revista de Educación a Distancia, 1-14.

Grisales, C. (2013). Implementación de la plataforma Moodle en la Institución Educativa Luis López de Mesa. Medellín, Colombia: Universidad Nacional de Colombia.

Guiffrida, D.; Lynch, M. \& Wall, A. A. (2013). Do Reasons for attending college affect academic outcomes? A test of a motivational model from a selfdetermination theory perspective. Journal of College Student Development, 1-20.

Hilbert, M. \& Cairó, O. (2009). Quo Vadis, Tecnología de Información y de las Comunicaciones. Colombia: Cepal.

Morales, C. (2012). El uso de la plataforma Moodle con los recursos web 2.0 y su relación con las habilidades del pensamiento crítico en el sector historia, geografía y ciencias sociales. Santiago de Chile: Universidad de Chile.

Muñoz, L.; Gómez, M. \& Alemán, L. (2016). Uso de la plataforma educativa Moodle en los procesos de 
capacitación de maestros de Educación Indígena en Jalisco, México. Revista del Instituto de Estudios en Educación Universidad del Norte. No 24, 28 - 42.

Rodríguez Conde, M. J. (2005). Aplicación de las TIC a la evaluación de alumnos universitarios. Teoría de la Educación. Educación y Cultura en la Sociedad de la Información, 8-25.
Rodríguez, A. (2010). Estudio, desarrollo, evaluación e implementación del uso de plataformas virtuales en entornos educativos en Bachillerato, ESO y programas específicos de atención a la diversidad: programas de diversificación curricular, programa de integración y programa. Madrid: Universidad Autónoma de Madrid. 